\title{
Psychological Distress, Persistent Physical Symptoms, and Perceived Recovery After COVID-19 IIIness
}

J Gen Intern Med 36(8):2525-7

DOI: $10.1007 / \mathrm{s} 11606-021-06855-\mathrm{w}$

(C) Society of General Internal Medicine 2021

\section{INTRODUCTION}

Many patients hospitalized for COVID-19 report physical and psychological symptoms that persist for months after viral clearance - the so-called "long-haulers". ${ }^{1}$ Symptom persistence is not only limited to those with severe forms of COVID19 , but also often occurs in patients with mild-to-moderate illness not requiring intensive care or prolonged hospitalization. ${ }^{2,3}$ COVID-19 can also result in symptoms of depression, anxiety, and/or posttraumatic stress disorder (PTSD), which may interfere with subsequent physical recovery. ${ }^{4}$ In this study, we examined the association of depression and PTSD with perceived recovery following COVID-19 illness.

\section{METHODS}

Adults hospitalized for COVID-19 at two Columbia University hospitals and discharged home between March 26 and May 27, 2020 were invited to complete a 3-month post-discharge survey assessing the physical and psychological impact of their COVID-19 illness. Surveys could be completed in English or Spanish via telephone or online. Survey questions included demographics (age, sex, race, ethnicity), history of mental illness (depression, anxiety, PTSD), and the presence of nine common COVID-19 physical symptoms both during and after hospitalization. PTSD was assessed using the PTSD Checklist for DSM-5, with questions asked in reference to the COVID-19 illness (PCL-5, scores $\geq 30$ considered positive). Depression was assessed using the 8-item Patient Health Questionnaire (PHQ-8, scores $\geq 10$ considered positive). COVID-19 recovery was determined by asking "Overall, how much have you recovered to your usual state before having COVID-19?" (0-not at all, 1-a little, 2-mostly, 3 - fully). Responses were coded " 1 " for fully recovered and " 0 " otherwise. Length of stay, level of inpatient care, and intubation during the COVID-19 hospitalization were obtained from the medical record. Logistic regression was used

Prior Presentations The current study was presented as an abstract at the American Psychosomatic Society 2020 Virtual Meeting held on December 3-4, 2020.

Received February 18, 2021

Accepted April 22, 2021

Published online May 13, 2021 to estimate the association of COVID-related PTSD and depression with perceptions of recovery, adjusting for covariates. Analyses were performed using SAS statistical software (v9.4, SAS Institute) at a two-sided alpha of 0.05 . The Columbia University Institutional Review Board approved the study, and all patients provided informed consent.

\section{RESULTS}

Of 510 eligible patients, 153 completed the survey ( 58 by telephone, 95 online, median 3.7 [range 2.6-5.7] months post-discharge). Mean age was $54.5 \pm 16.7$ years, $39.9 \%$ were female, $17.0 \%$ were White, $15.0 \%$ were Black, $54.9 \%$ were Hispanic, and $15.0 \%$ reported a history of mental illness (13.1\% depression, $8.5 \%$ anxiety, $2.6 \%$ PTSD, $2.0 \%$ other). The mean length of COVID-19 hospitalization was $8.0 \pm 8.1$ days, $5.9 \%$ of patients required ICU admission, and $4.6 \%$ were intubated. Only $35.5 \%$ of patients reported full recovery from their COVID-19 illness, 23.5\% had COVID-related PTSD, $18.3 \%$ had depression, and $12.4 \%$ met the criteria for both.

The most common persistent COVID-related physical symptoms were body aches (23.5\%), fatigue (20.3\%), shortness of breath (19.0\%), and headaches (13.1\%), each of which was more likely to be reported by patients with depression or PTSD (Fig. 1). Depression and PTSD were each associated with a greater mean number of persistent physical symptoms (depression: 2.46 vs. $0.73, p<0.001$; PTSD: 2.08 vs. 0.69 , $p<0.001$ ) and a higher likelihood of feeling unrecovered (depression: $85.7 \%$ vs. $60.5 \%$, OR $3.92,95 \%$ CI $1.28-11.99$, $p=0.02$; PTSD: $91.7 \%$ vs. $56.5 \%$, OR 8.46 , $95 \%$ CI $2.45-$ $29.18, p<0.001)$. In the fully adjusted model, this association became non-significant for depression (OR 3.12, 95\% CI $0.51-19.12, p=0.22)$ but remained borderline significant for PTSD (OR 4.93, 95\% CI 0.86-28.37, $p=0.07$; Table 1).

\section{DISCUSSION}

Patients with COVID-related PTSD and depression had a higher burden of persistent physical symptoms and were less likely to feel fully recovered 3 months after their COVID-19 illness. PTSD and depression have been associated with interoceptive dysfunction, which may perpetuate physical symptoms long after the acute illness has resolved. ${ }^{5,6}$ Therefore, one interpretation of our findings is that patients with PTSD and/or depression are more sensitive to physical symptoms, 

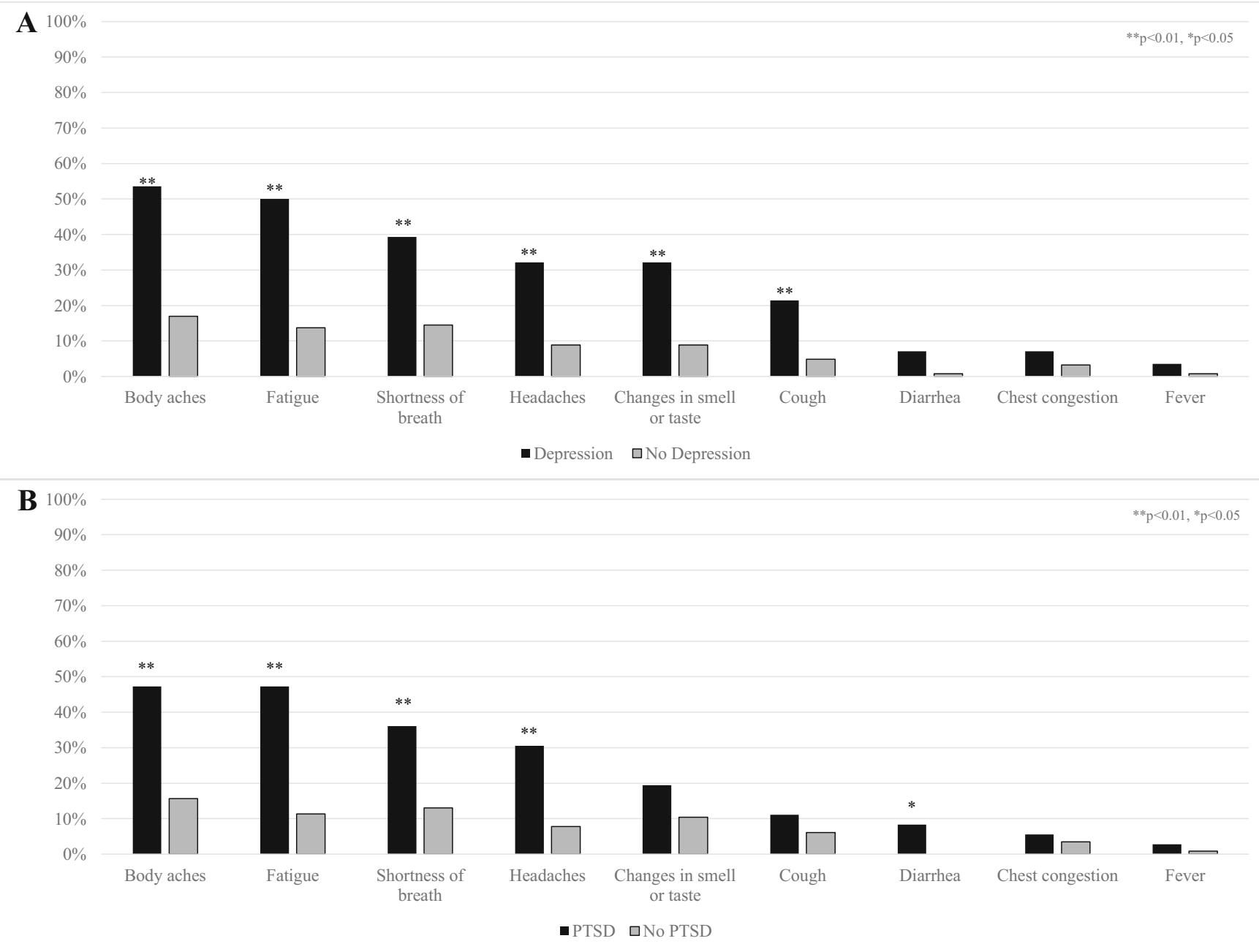

Figure 1 Prevalence of persistent COVID-related physical symptoms 3 months after COVID-19 hospitalization according to A. depression status (PHQ-8 score $\geq 10$ vs. PHQ-8 score $<10$ ) and B. PTSD status (PCL-5 score $\geq 30$ vs. PCL-5 score $<30$ ); $n=153$.

experience heightened psychological distress in response, and consequently feel less recovered from their COVID-19 illness.

Study limitations included single-center recruitment from a predominantly hospitalized, urban, Hispanic population, thereby reducing generalizability; modest sample size with limited power to detect clinically meaningful differences; and low survey completion rates subject to potential response

Table 1 Association of Patient Demographic and Clinical Variables with Perceptions of Recovery Following COVID-19 Hospitalization $(n=$ 153)

\begin{tabular}{|c|c|c|c|c|c|c|}
\hline Characteristic & Unadjusted OR & $95 \% \mathrm{CI}$ & $p$-value & Adjusted OR & $95 \% \mathrm{CI}$ & $p$-value \\
\hline Age (per 1-year increase) & 0.99 & $0.97-1.02$ & 0.61 & 1.00 & $0.97-1.04$ & 0.86 \\
\hline Female (vs. male) & 1.33 & $0.67-2.65$ & 0.42 & 0.69 & $0.23-2.07$ & 0.51 \\
\hline Black (vs. non-Black) & 2.18 & $0.76-6.24$ & 0.15 & 3.46 & $0.61-19.68$ & 0.16 \\
\hline Hispanic (vs. non-Hispanic) & 0.68 & $0.35-1.33$ & 0.26 & 0.93 & $0.29-2.95$ & 0.90 \\
\hline Length of hospitalization ${ }^{*}$ & 1.03 & $0.97-1.09$ & 0.35 & 0.99 & $0.93-1.07$ & 0.86 \\
\hline Self-reported mental illness ${ }^{\dagger}$ (vs. none) & 1.31 & $0.50-3.42$ & 0.59 & 0.85 & $0.18-3.92$ & 0.83 \\
\hline Depression & 3.92 & $1.28-11.99$ & 0.02 & 3.12 & $0.51-19.12$ & 0.22 \\
\hline COVID-induced PTSD ${ }^{\S}$ & 8.46 & $2.45-29.18$ & $<0.001$ & 4.93 & $0.86-28.37$ & 0.07 \\
\hline Persistent physical symptoms & 2.72 & $1.65-4.46$ & $<0.001$ & 4.37 & $1.79-10.65$ & 0.001 \\
\hline
\end{tabular}

Abbreviations: PTSD, posttraumatic stress disorder; PHQ-8, Patient Health Questionnaire (8-item); PCL-5, PTSD Checklist for DSM-5

*Days hospitalized for COVID-19 illness

tDepression, anxiety, PTSD, and/or other

$\pm P H Q-8$ score $\geq 10$ vs. $P H Q-8$ score $<10$

PCL-5 score $\geq 30$ vs. PCL-5 score $<30$

"Sum of persistent COVID-19 physical symptoms (range 0-9)

"Bivariate analyses examining the unadjusted associations between each of the listed variables and perceived COVID-19 recovery

${ }^{\#}$ Logistic regression model including all listed variables to estimate their adjusted associations with perceived COVID-19 recovery 
bias. Nevertheless, our estimates of symptom persistence are consistent with prior studies. ${ }^{2}$

Notwithstanding these limitations, this is the first study to examine the association between psychological distress and COVID-19 recovery. Our findings highlight the importance of mental health screening in the management of post-acute COVID-19. Furthermore, they suggest that addressing depression and PTSD may be needed to optimize psychological and physical recovery from COVID-19.

Nadia A. Liyanage-Don, $M D, M S^{1}$

Talea Cornelius, $\mathrm{PhD}, \mathrm{MSW}^{1}$

Jose E. Sanchez, BS ${ }^{2}$

Alison Trainor, $M S^{1}$

Nathalie Moise, $M D, M^{1}$

Milton Wainberg, $M D^{3,4}$

Ian $M$. Kronish, $M D, M P H^{1}$

${ }^{1}$ Center for Behavioral Cardiovascular Health, Columbia University Irving Medical Center, New York, NY, USA

${ }^{2}$ Vagelos College of Physicians and Surgeons, Columbia University Irving Medical Center, New York, NY, USA

${ }^{3}$ Department of Psychiatry, Columbia University Irving Medical Center,

New York, NY, USA

${ }^{4}$ New York State Psychiatric Institute, Columbia University Irving Medical Center,

New York, NY, USA

Corresponding Author: Ian M. Kronish, MD, MPH; Center for Behavioral Cardiovascular Health, Columbia University Irving Medical Center, New York, NY, USA (e-mail: ik2293@cumc.columbia.edu).

Author Contributions IMK conceived and designed the study; supervised the study protocol, participant recruitment, and data collection; and obtained research funding. NM and MW provided advice on study design and methodology. TC provided advice on statistical analysis. AT and JES helped to coordinate the study and curate the collected data. NAL contributed to the study design, analyzed the data, and drafted the manuscript. All authors contributed substantially to manuscript revision. IMK takes responsibility for the paper as a whole.

Funding Dr. Liyanage-Don received funding support from the Health Resources and Services Administration Primary Care Training and Enhancement Program (TOBHP29302).Dr. Cornelius received funding support from the National Center for Advancing Translational Sciences (KL2TRO01874) and the National Institute on Aging (U24AG052175).Mr. Sanchez received funding support from the National Heart, Lung, and Blood Institute (T35HL007616).Dr. Moise received funding support from the National Heart, Lung, and Blood Institute (R01HL141609) and the Agency for Healthcare Research and Quality (RO1HSO25198).Dr. Wainberg received funding support from the National Institute of Mental Health (T32MH096724, U19MH113023).Dr. Kronish received funding support from the $\mathrm{Na}$ tional Heart, Lung, and Blood Institute (RO1HL123368, R01HL132347) and the National Institute on Aging (P30AG064198, U24AG052175).

\section{Declarations:}

Conflict of Interest: The authors do not have any conflicts of interest to declare.

Disclaimer: The study sponsor had no role in the study design; collection, analysis, or interpretation of the data; preparation of the manuscript; or decision to submit the manuscript for publication.

\section{REFERENCES}

1. Rubin R. As Their Numbers Grow, COVID-19 "Long Haulers" Stump Experts. JAMA. 2020 Oct 13;324(14):1381-1383.

2. Goërtz YMJ, Van Herck M, Delbressine JM, et al. Persistent symptoms 3 months after a SARS-CoV-2 infection: the post-COVID-19 syndrome? ERJ Open Res. 2020 Oct 26;6(4):00542-2020.

3. Carvalho-Schneider C, Laurent E, Lemaignen A, et al. Follow-up of adults with noncritical COVID-19 two months after symptom onset. Clin Microbiol Infect. 2021 Feb;27(2):258-263.

4. Hossain MM, Tasnim S, Sultana A, et al. Epidemiology of mental health problems in COVID-19: a review. F1000Res. 2020 Jun 23;9:636.

5. McFarlane AC, Weber DL, Clark CR. Abnormal stimulus processing in posttraumatic stress disorder. Biol Psychiatry. 1993 Sep 1;34(5):311-20.

6. Paulus MP, Feinstein JS, Khalsa SS. An Active Inference Approach to Interoceptive Psychopathology. Annu Rev Clin Psychol. 2019 May 7;15:97122 .

Publisher's Note: Springer Nature remains neutral with regard to jurisdictional claims in published maps and institutional affiliations. 